\title{
Numerical simulation of resistivity logs in complex three-dimensional fractured car- bonate reservoirs using finite elements and integral equation approaches
}

\author{
Alfredo Carrasco ${ }^{1}$ \& Abel Carrasquilla ${ }^{2},{ }^{1}$ UFF/TEQ, Niterói - RJ \& ${ }^{2}$ UENF/LENEP, Macaé - RJ
}

Copyright 2016, SBGf - Sociedade Brasileira de Geofísica

Este texto foi preparado para a apresentação no VII Simpósio Brasileiro de Geofísica, Ouro Preto, 25 a 27 de outubro de 2016. Seu conteúdo foi revisado pelo Comitê Ouro Preto, 25 a 27 de outubro de 2016. Seu conteúdo foi revisado pelo Comitê
Técnico do VII SimBGt, mas não necessariamente representa a opinião da SBGf ou de seus associados. É proibida a reprodução total ou parcial deste material para propósitos comerciais sem prévia autorização da SBGf.

\begin{abstract}
This study simulates the response of deep and medium resistivity induction logs in fractured carbonate reservoirs using the integral equation numerical technique. We used as a base the Miranda et al. (2009) model, whose laterolog resistivity results were verified using the Cozzolino (2004) finite element algorithm. Using the piston-type mud invasion, our resistivity induction results indicate that when the invasion is $0.5 \mathrm{~m}$, there is nearly no difference between both curves. Still, when the mud intrusion is increased to 1.0 and $1.5 \mathrm{~m}$, a bigger separation between them gets up, which shows a tight link between the invasion and permeability.
\end{abstract}

\section{Introduction}

Since induction (IL) tools were introduced, log analysts and petrophysicists have been faced with the hard decision of which resistivity tool to run: laterolog (LL) or IL. Both logging tools each has unique features that favor their use in specific situations and applications and there have been recommendations and guidelines from long ago to the present, showing different factors to consider when choosing and interpreting resistivity logs. Nevertheless, one of the most important components to consider, which is sometimes overlooked, is the dividing line between the flushed zone (Rxo) and formation (Rt) resistivity.

IL measurements respond to conductivity and they are considered conductivity-sensing devices. LL measurements respond to resistivity, and, therefore, they are resistivity devices. In this manner, when Rxo>Rt, Rxo strongly influences the measurements because attenuates little the applied magnetic field, but hinders the passage of electric current, being good conditions for IL tool. On the opposite, when Rt>Rxo, a LL tool is preferred for Rt determination, cause the conductivity in the borehole facilitates the passage of electric current and mitigates the applied magnetic field. Therefore, IL tools are best fitted for low to medium-resistivity formations and recommended for wells drilled with only moderately conductive drilling muds or nonconductive muds (e.g., oil-based mud) and for air-drilled holes. LL, on the other hand, is a resistivity tool that is best used in the opposite conditions: they are more accurate in medium- to high-resistivity formations and recommended for wells drilled with very conductive muds (i.e., salt muds). In practice, both modern LL and IL logging tools are suitable for most logging conditions, and it is no longer practical to make a specific recommendation for one type in preference to the other, except in extreme conditions (Anderson, 2001).

Exploration and evaluation programs for carbonates are similar to those for sandstone reservoirs. Nonetheless, new instruments, techniques, and interpretation methodologies are needed to direct the specific challenges above to drill and produce these reservoirs optimally (Schlumberger, 2001). In this study, we simulate Rt curves in conditions where there is a drilling mud filtrate invasion taking place in complex fractured reservoirs through numerical modeling using the integral equation technique. In thus doing, we numerically simulate dual - LL and array IL resistivity logs to validate the estimation of petrophysical properties.

\section{Methodology}

In our study, the reservoir representation was made on a smaller scale, since we are only simulating tool responses which have a given radius of investigation, within a representative petrophysical model. Thus, we simulated the LL and IL log responses of a carbonate reservoir model containing faults, fractures and bugs vugs (Figure 1), functioning as a reference to a typical carbonate reservoir (Figure 2). In this sense, authors as Erketin et al. (2001) proposed models considering a radial flow as shown in Figure 3, which could also be represented as shown in Figure 4, where the thick lines would represent the fracture system. And then, taking this representation as reference, the model is calibrated on the basis a well log obtained in a carbonate reservoir, in society to determine up the parameters of the radial model.

To make the study, initially, we simulated the LL responses (Anderson, 2001) adopting a code developed by Cozzolino (2004) which use the finite element numerical technique and models with axial symmetry (Figure 5). To fine-tune our models, the model of Miranda et al. (2009) for the Venezuelan wellbore BOR-11 was used (Figure 6), which is characterized by the presence of vugs and fractures, obtaining laterolog deep (LLD) and shallow (LLS) logs. Both results were compared, and once this first step has been overcome after the evidence that our results are correct, it is possible to move on to different simulations for different depths of invasion using the Cozzolino (2004) integral equation approach to simulate IL responses with different geometrical characteristics of a carbonate reservoir. In practice, little is known regarding the actual invasion geometry and there are no satisfactory ways of measuring them in situ. A simplified model widely used for the determination of $\mathrm{Rt}$ in this case is the piston type, which can be described as a uniform displacement of the original formation fluid by the drilling fluid (washed zone 
with resistivity called $\mathrm{R} \times 0$ ). In all instances were considered a wellbore radius of $0.1 \mathrm{~m}$ and mud resistivity of 2 ohm-m.

\section{Results}

The responses of the adjustment of geometrical parameters of Figure 6, based on the results obtained after doing the simulation of LLS and LLD resistive curves in Venezuelan well BOR-11 with a carbonate reservoir (Figure 7), are represented in the Figure 8 , giving as responses a fracture system spaced $25 \mathrm{~cm}$ from each other, and each with a clearance of $3 \mathrm{~mm}$, making the observation that this system would not only represents a system of fractures, but also the presence of bugs vugs, as indicated initially. Notice that fractures are filled with drilling fluid resistivity value. In this figure the fractures appear represented by a gray color. Based on this model, we made some simulations of the responses of the deep (ILD) and medium (ILM) induction logs, considering a well diameter of $0.1 \mathrm{~m}$ and a mud resistivity of 2 ohms.m.

As In a first example, we establish a simulation without considering mud-cake, since the tendency of IL is to make deeper readings, and without invasion, in order to compare the responses obtained between fractured and no fractured systems. In this example we are considering a rock matrix resistivity of $1000 \mathrm{ohm}$. $\mathrm{m}$ and fractures resistivity of 2 ohm.m as a result of drilling fluid entries. The thickness of the layer of interest is $2.512 \mathrm{~m}$ and limited in top and bottom by shale with $1 \mathrm{ohm}$.me of resistivity value. It was considered that the radial extent of the fracture system is $1.768 \mathrm{~m}$. The results can be seen in Figure 9, showing the ILD responses in red color and the ILM responses in green color, with the dashed lines corresponding to the reservoir containing fractures. In this figure we see just about differences between the responses of a reservoir containing or not fractures, and then we would have a capillary invasion of a conductive fluid in a resistive medium. If the drilling fluid were electrically resistive, little variation between these responses would be anticipated. We also noted the decrease in the resistivity value of the geoelectrical model caused by the presence of conductive fluid.

In the second simulated case, we applied the same model described before, but now considering a piston type invasion of $0.5 \mathrm{~m}$ length and having a resistivity of $50 \mathrm{ohm}$. m. In the same figure it was placed a model response without invasion, for comparison purposes, as indicated in Figure 10. In this figure, red lines represent ILD and blue lines represent ILM. The dashed lines represent the responses that consider invasion. In the same figures we observe that the simulated response ILD (in red color) is almost coincident with the response without invasion, a fact that is expected because of the length of depth invasion about 0.5 meters.

In the third and the fourth case, in addition to fracture systems, we simulated a piston type invasion with a distance of $1.0 \mathrm{~m}$ and $1.5 \mathrm{~m}$ each other, with the same invaded zone resistivity value of the previous case (50 ohm. $\mathrm{m})$, as shown in Figures 11 and 12. In these two figures we note that when the extent of invasion is increased, there is a greater separation between the continuous and dashed curves for each simulated case.

\section{Conclusions}

This study shows the results of the numerical simulation of resistivity logs from the carbonate reservoir of South Wasson Clear Fork, based on study data made in the well BOR-11. The results of the modeling with the algorithm developed by Cozzolino (2004), which employs a finite element technique, show a good fit with the responses of the LLD and LLS laterolog tools obtained by Miranda et al. (2009). After that, we use the same algorithm, but now simulating the ILD and ILM induction logs for different length invasion. The results indicate that when the invasion is increased, there is a greater separation between deep (continuous) and medium (dashed) curves for each simulated situation. This indicates a close connection between the invasion and permeability, that is, when there is a greater invasion in the reservoir it clearly indicates that there is a greater permeability.

\section{Acknowledgements}

We thank to UENF by the computational support, the SPE by providing articles for consultation and Petrobras Science, Training and Technology in Carbonates (SCTC) by the financial support of the research project.

\section{References}

Anderson, B. 2001. Modeling and inversion methods for the interpretation of resistivity logging tool response. PhD Thesis, DUT -Deft, 386 pp.

Cozzolino, K. 2004. Study of transient temperature effects and invasion in well logs. PhD Thesis, UFRJ - Rio de Janeiro, 146 pp. (In Portuguese).

Erketin, T.; Abou-Kassem, J. \& King, G. 2001. Basic applied reservoir simulation. Society of Petroleum Engineers, $406 \mathrm{pp}$.

Miranda, L.; Torres, C. \& Lucia, J. 2009. Modeling mudfiltrate invasion effects on resistivity logs to estimate permeability of vuggy and fractured carbonate formations. SPE 121136.

Schlumberger. 2013. Log interpretation charts. Educational Services. USA, $306 \mathrm{p}$. 


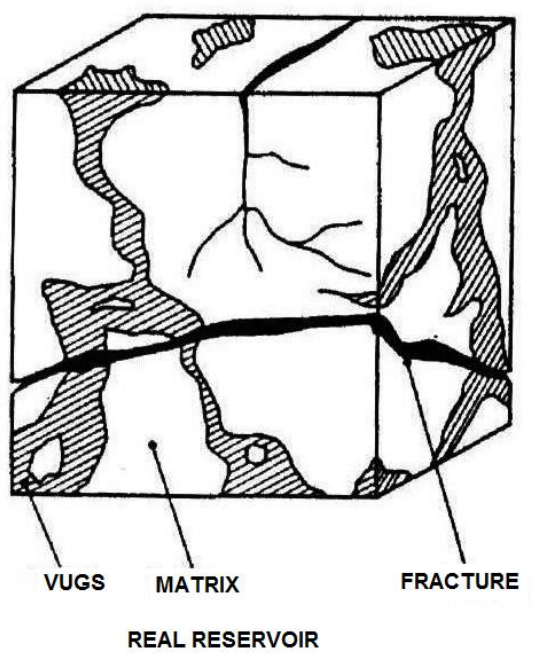

Figure 1. Carbonate reservoir scheme (from Erketin et al., 2001).

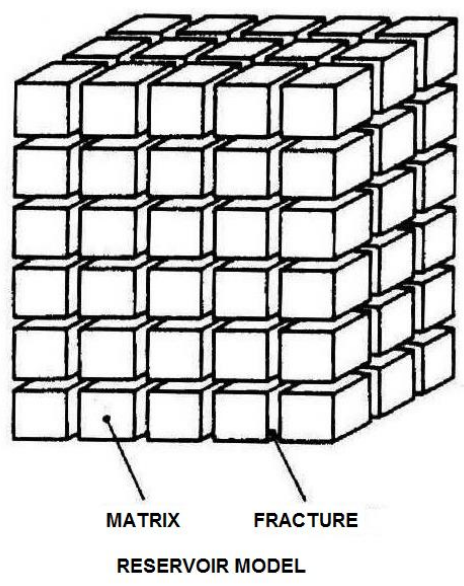

Figure 3. Carbonate reservoir scheme (from Erketin, 2001).

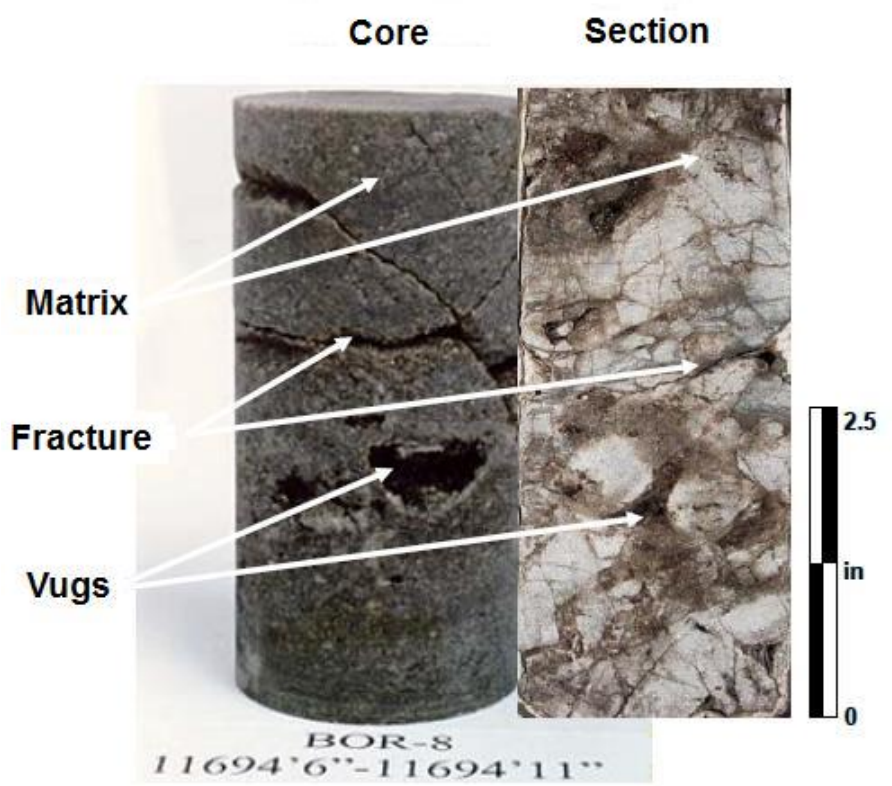

Figure 2. Carbonate core and section (from Miranda et al, 2009).

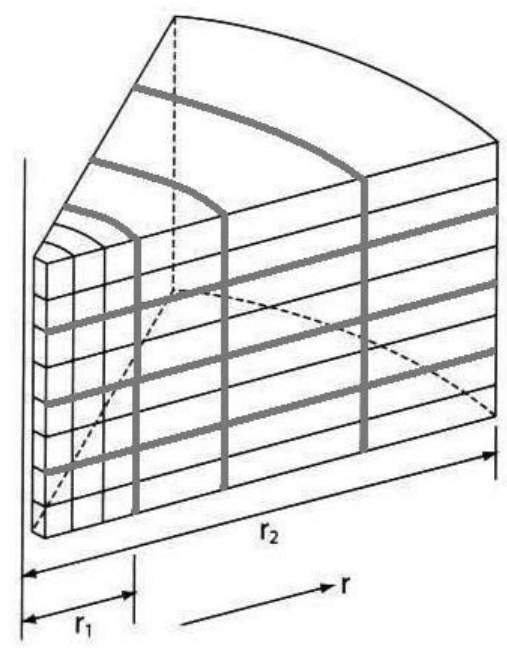

Figure 4. Radial representation of a fractured system (from Erketin, 2001). 


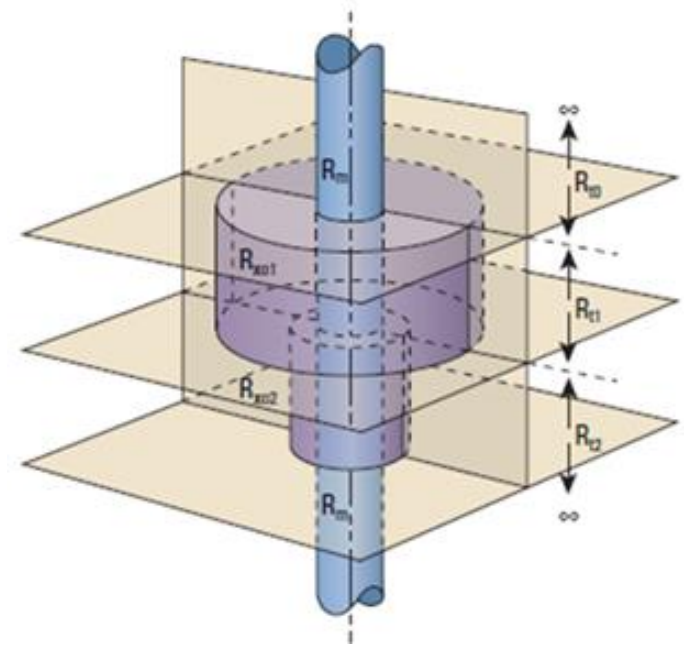

Figure 5. Axial or 2.5D three dimensional model around the borehole (modified from Anderson, 2004)
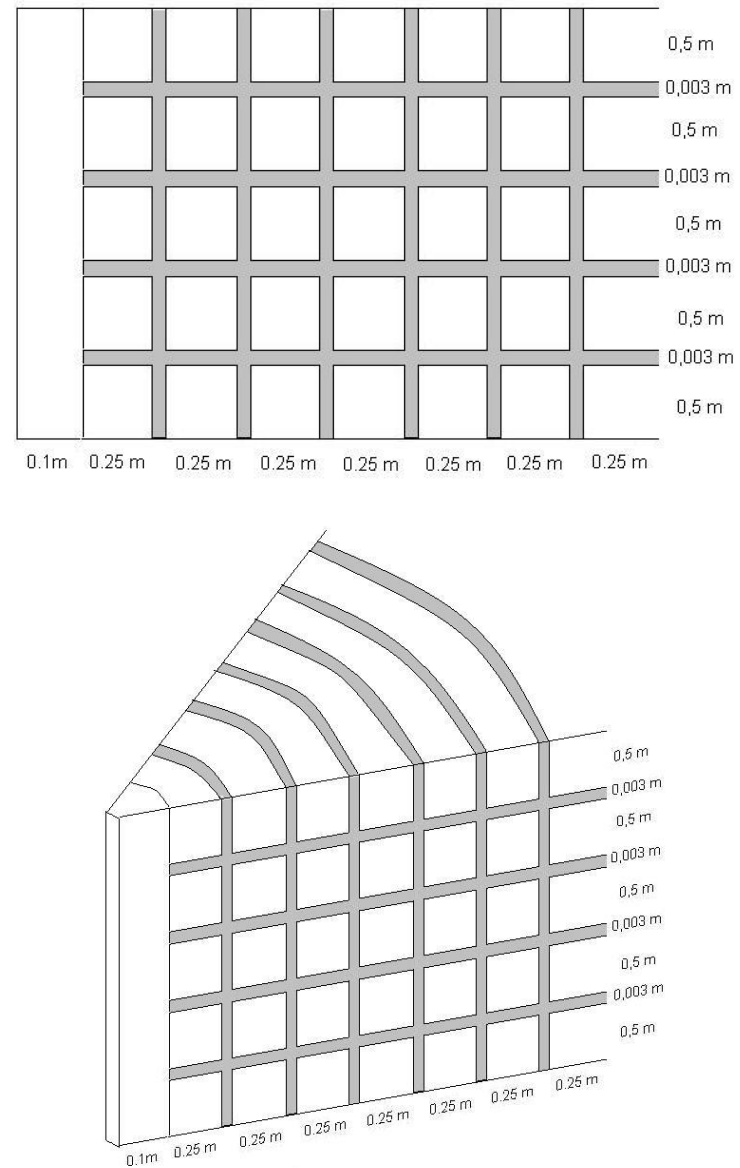

Figure 6. Axial view of the reservoir model for the wellbore BOR-11.

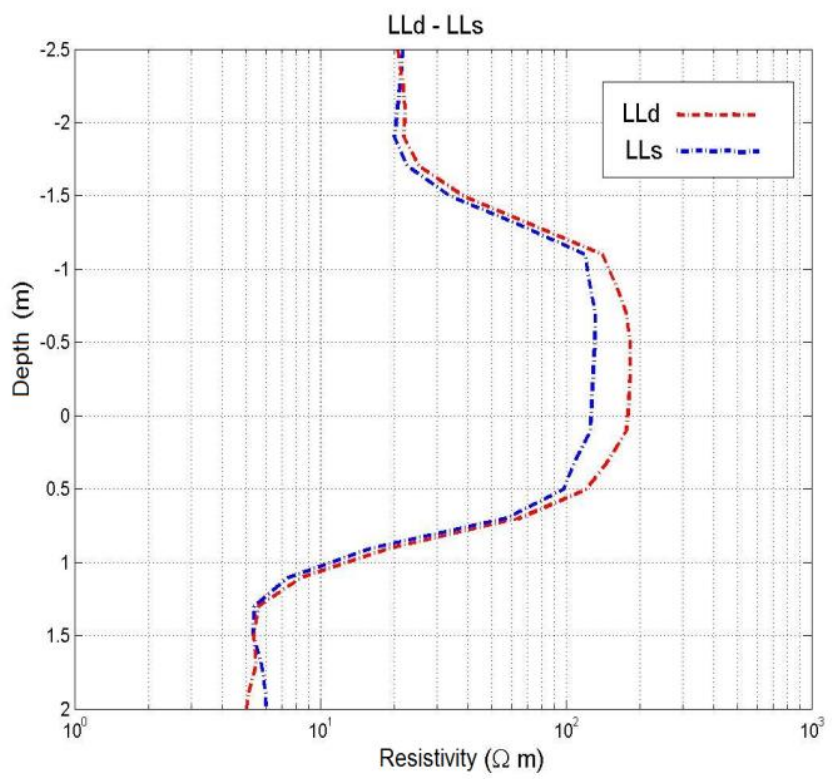

Figure 8. Laterolog responses from the wellbore BOR-11 Venezuela using Cozzolino (2004) algorithm.
Figure 7. Laterolog responses from the wellbore BOR-11 Vene-

zuela (from Miranda et al., 2009).

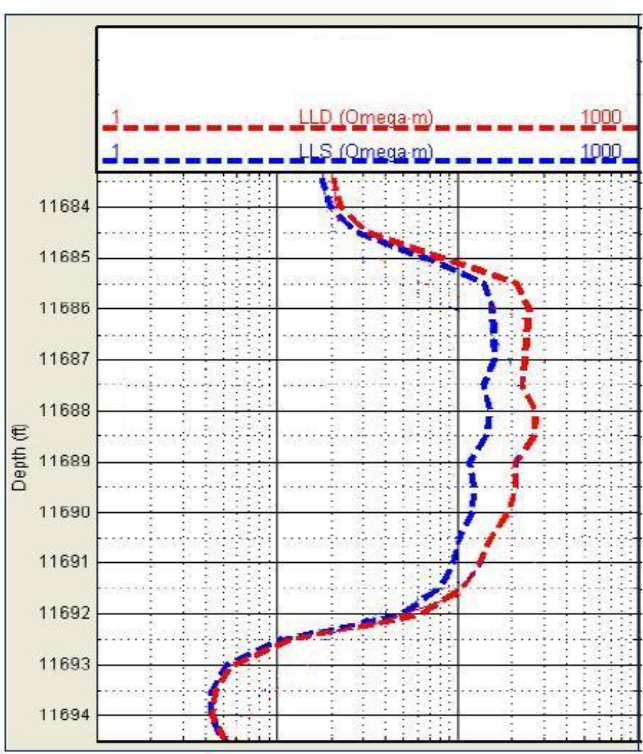




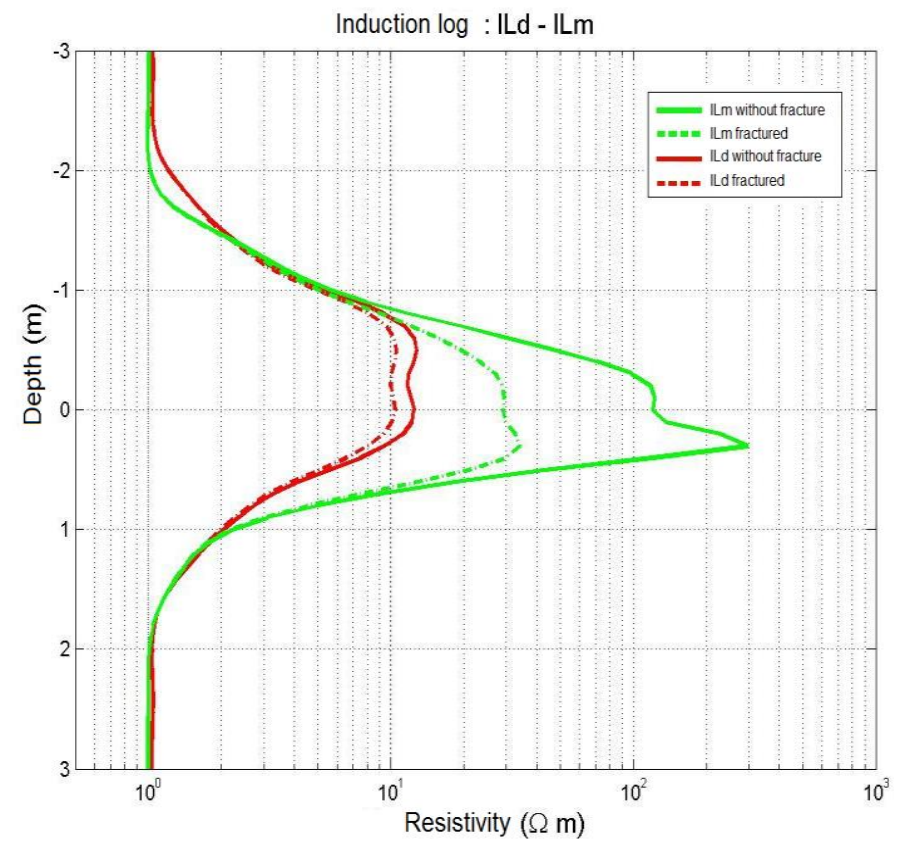

Figure 9. Responses of the carbonatic model without invasion.

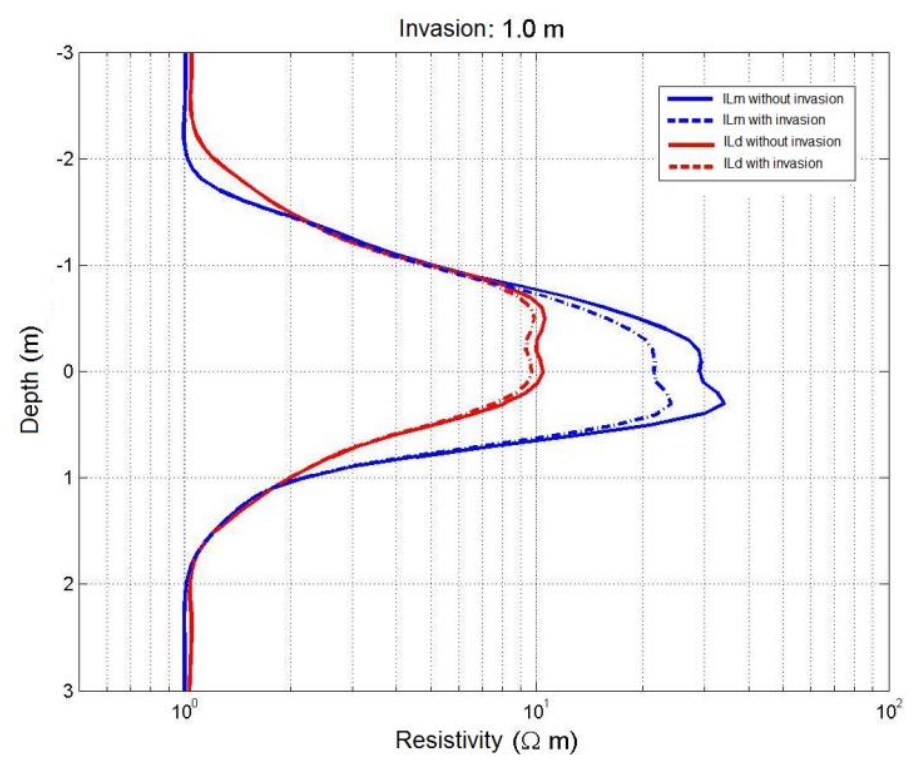

Figure 11. ILD and ILM simulation with an invasion depth of $1.0 \mathrm{~m}$.

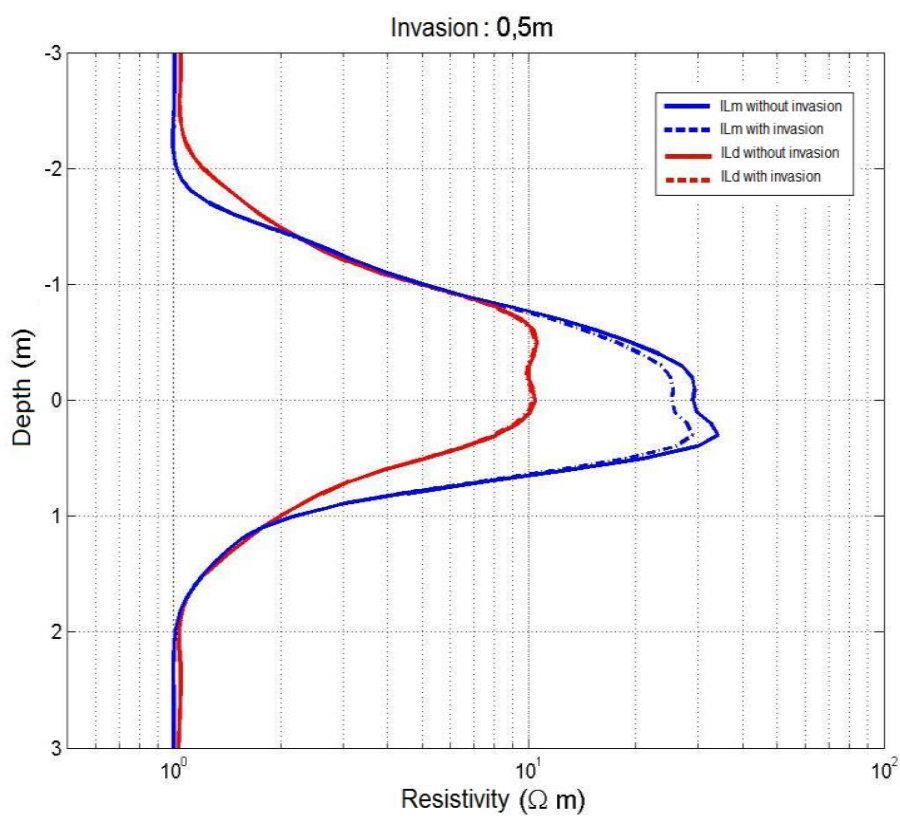

Figure 10. ILD and ILM simulations with an invasion depth of $0.5 \mathrm{~m}$.

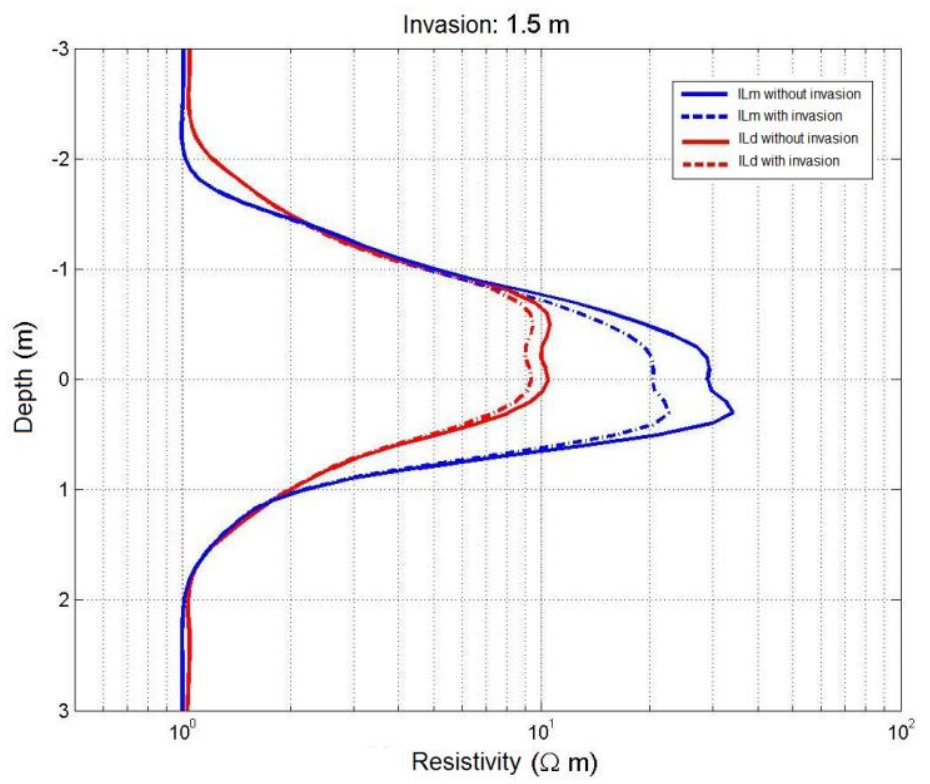

Figure 12. ILD and ILM simulation with an invasion depth of $1.5 \mathrm{~m}$. 